\title{
X-ray variability of accreting black hole systems: propagating-fluctuation scenario
}

\author{
Patricia Arévalo, Phil Uttley and Ian McHardy \\ University of Southampton, Southampton, SO17 1DH, UK \\ email: patricia@astro.soton.ac.uk
}

\begin{abstract}
Propagating fluctuation models can reproduce fundamental properties of the variability observed in the X-ray light curves of accreting black hole systems. We explore this type of model and show how extended emitting regions introduce at the same time energy dependent power spectral densities (PSD) and time lags between different energy bands.
\end{abstract}

Keywords. Galaxies: active - X-rays: binaries, galaxies - accretion disks

X-ray light curves from AGN and XRBs show fluctuations on a very broad range of timescales, which is hard to reconcile with the compact nature expected for the X-ray emitting region. Lyubarskii (1997) proposed a solution where fluctuations in the accretion rate produced at different radii in the accretion flow propagate inward, to finally modulate the emission at the central, X-ray emitting region.

Propagating fluctuations produce a broad range of variability timescales and higher variability amplitudes at higher flux levels. Following Lyubarskii (1997), we assumed that independent fluctuations are introduced at geometrically spaced radii, with a radiallydependent characteristic timescale and that they propagate inward with viscous velocity (Arévalo \& Uttley 2006). Light curves produced numerically following this procedure (Fig. 1a) have a $\mathrm{PSD} \propto 1 / f$ (Fig. 1b) with a high frequency cutoff, as observed in many AGN and XRBs in the high/soft state. The method also reproduces the linear relation between rms variability and flux (Fig. 1c) seen in both types of objects (Uttley \& McHardy 2001).

Different emissivity profiles for different energy bands produce energy-dependent power spectra and time lags between the bands. Observationally, softer X-ray light curves show less variability on short timescales and tend to lead the variability of harder bands. This behaviour can be achieved by the model if the energy spectrum of the emitting region hardens towards the centre, i.e. if the softer X-rays are emitted by a more extended region. Figure 2a shows the PSDs of two simulated light curves, which differ only by the extent of their emitting regions, the solid line corresponds to a radial emissivity profile $\epsilon(r) \propto r^{-3.5}$ and the dots correspond to $\epsilon(r) \propto r^{-3}$. The flatter the emissivity profile, the more high frequency variability power is suppressed. Also, as the fluctuations propagate towards the central black hole, they are seen first in the more extended emitting region (soft) and later in the more concentrated emitting region (hard), which produces hard time-lags, that increase both with the timescale of the fluctuations and with the difference in emissivity indices. The lag spectrum calculated between the simulated light curves that produce the PSDs in Fig. 2a is shown in Fig. $2 b$.

PSDs showing peaks can be produced by the propagating fluctuation model if only a few radii contribute to the variability. The effect of the extended emitting region is to create a stepped lag spectrum (Fig. 2b), from the lag value associated with one of these radii to the lag of the next. This might be the case in the Seyfert 1 galaxy Ark 564 (Fig. 2c) where the PSD appears to contain two components (tentatively shown by dotted and 

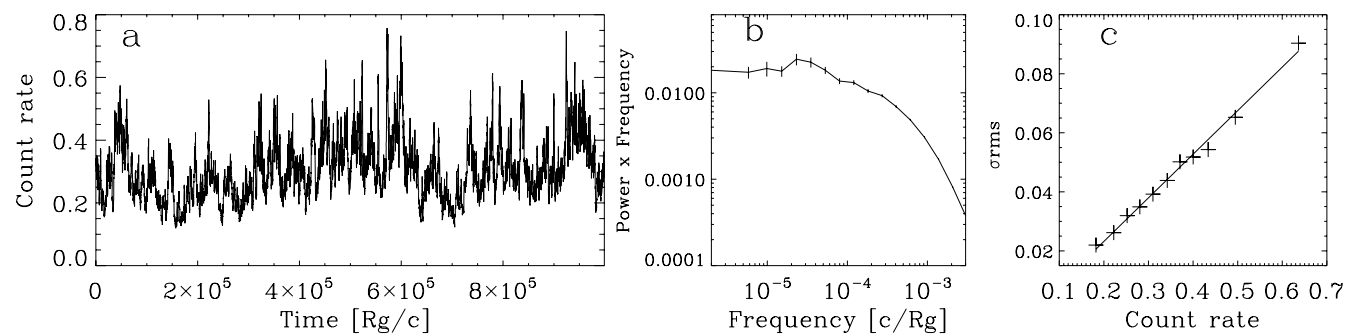

Figure 1. Simulated light curve (a), its Power spectrum (b) in terms of Power $\times f$, shows a flat top (corresponding to Power $\propto 1 / f$ ), that cuts off at high frequencies due to the filtering effect of an extended emitting region. Simulated light curves also reproduce the rms-flux relation (c).
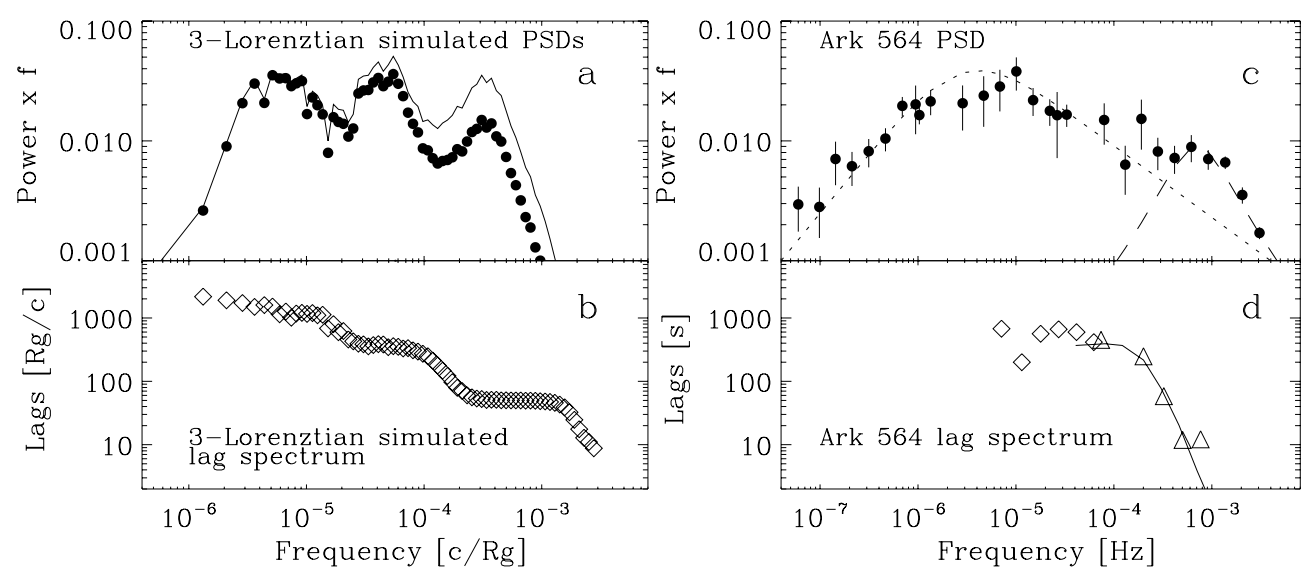

Figure 2. PSD (a) and lag spectrum (b) of two simulated light curves produced by assuming only three annuli in the accretion flow contribute to the variability. The light curves are calculated with different emissivity profiles (see text) which introduce the difference in the high frequency power and the time lags. PSD (c, from McHardy et al. in prep) and lag spectrum (d, from Arévalo et al. 2006) of the Seyfert 1 galaxy Ark 564. The PSD shape suggests two main variability components, which is supported by the marked drop in the lag spectrum at the frequency where the components overlap.

dashed lines), and the lag spectrum shows a marked drop at the frequency where the components overlap (Fig. 2d).

The general propagating fluctuation model can reproduce several observed variability properties, PSD shape and energy dependence, rms-flux relation and time lags. This approach connects the energy dependence of the PSD with the lag spectrum, so both measurements can be used jointly to disentangle different components of the variability.

\section{References}

Arévalo, P. \& Uttley, P. 2006, MNRAS 367, 801

Arévalo, P., Papadakis, I., Uttley, P., McHardy, I. \& Brinkmann, W. 2006, MNRAS 372, 401

Lyubarskii, Y. 1997, MNRAS 292, 679

Uttley, P. \& McHardy, I. 2001, MNRAS 323, 26 